\title{
Speckle Suppression in Ultrasonic Images Based on Undecimated Wavelets
}

\author{
Fabrizio Argenti \\ Dipartimento di Elettronica e Telecomunicazioni, Università di Firenze, Via di Santa Marta 3, 50139 Firenze, Italy \\ Email:argenti@lenst.det.unifi.it \\ Gionatan Torricelli \\ Dipartimento di Elettronica e Telecomunicazioni, Università di Firenze, Via di Santa Marta, 3, 50139 Firenze, Italy \\ Email: torricelli@det.unifi.it
}

Received 11 February 2002 and in revised form 29 October 2002

\begin{abstract}
An original method to denoise ultrasonic images affected by speckle is presented. Speckle is modeled as a signal-dependent noise corrupting the image. Noise reduction is approached as a Wiener-like filtering performed in a shift-invariant wavelet domain by means of an adaptive rescaling of the coefficients of an undecimated octave decomposition. The scaling factor of each coefficient is calculated from local statistics of the degraded image, the parameters of the noise model, and the wavelet filters. Experimental results demonstrate that excellent background smoothing as well as preservation of edge sharpness and fine details can be obtained.
\end{abstract}

Keywords and phrases: ultrasound image denoising, speckle filtering, linear minimum mean square error filtering, undecimated discrete wavelet transform.

\section{INTRODUCTION}

Since the introduction of first coherent imaging systems, speckle noise has been widely studied. Speckle makes a homogeneous object to assume a granular appearance, and consequently, the contrast of the image is drastically reduced. The presence of a speckle pattern in a coherently formed image is due to the received backscatter signal from unresolvable particles constituting the inspected mean. Particular attention has been reserved to speckle noise in ultrasonic images since the degradation in the acquired image implies strong uncertainties in the detection of pathologies performed by an expert human observer. The texture of the speckle pattern tends also to hide fine details useful for computer-aided diagnosis. Moreover, it severely decreases the effectiveness of image postprocessing algorithms.

The theoretical foundations of speckle were given in optics, where laser holographic image formation has been studied [1]. By using a laser as a monochromatic coherent radiation, it was possible to reconstruct the inspected object by using the backscattered signal. The signal statistical properties obtained by theoretical analysis have been validated in many other imaging systems using coherent radiation, like radar and ultrasound, even if, in these cases, the representation of the image obtained by envelope detection is poorer due to the propagation of the radiation through an inhomogeneous medium. Ultrasound images represent the worst case since the ultrasonic wave encounters multiple interfaces that implies a masking effect for those reflectors laying farther from the probe. Both phase and amplitude (speckle) noise degrade the backscattered signal. Phase aberration may occur because of the imperfections of the focusing system that is realized by means of a delay line for each transducer of the phased array. An additional contribute to this kind of aberration is given by the random delays generated while the ultrasonic wave propagates through regions with different density. A great variety of techniques have been devised to reduce the effect of the phase distortion $[2,3,4,5]$. Speckle noise, however, represents the principal cause of the whole degradation. In order to enhance the quality of the ultrasonic image, many different approaches have been proposed. Most of them can be related to averaging uncorrelated samples. In particular, effective results have been obtained with spatial compounding [6] and frequency compounding [7] that allow us to trade SNR improvement for loss of resolution in the lateral or longitudinal direction. Another algorithm based on frequency diversity has been proposed in [8]. A practical implementation of frequency diversity, based on split spectrum processing (SSP), has been introduced in [9]. Even if simple versions of these methods are currently used in many commercial ultrasonic systems, some other postprocessing algorithms have been developed to overcome the limit of resolution and overall 
system complexity imposed by compounding. For example, an adaptive median filter driven by local statistics is proposed in [10].

The proposed algorithm is based on spatial filtering applied in the wavelet domain. Several denoising algorithms based on the wavelet decomposition have been presented in the literature for additive signal-independent noise. Thresholding of wavelet coefficients was proposed by Donoho [11]. Wavelet thresholding adapted to the local context of the image has been presented in [12]. A Wiener-like approach working in the wavelet domain has been proposed in [13]. In [14], spatially adaptive rescaling based on a statistical model of wavelet coefficients was used.

Speckle noise, however, is not modeled as an additive signal-independent noise but as an additive signal-dependent noise. Models for ultrasonic speckle noise are discussed in Section 2. A recent approach working in a transformed domain to remove signal-dependent noise is presented in [15].

In this paper, we propose a denoising method based on linear minimum mean square error (LMMSE) estimation of wavelet coefficients. The observed wavelet coefficients are rescaled according to a coefficient computed, taking into consideration the wavelet filters, the noise-model parameters, and local statistics of the observed image. We use an undecimated wavelet decomposition, the advantages of which for smoothing signal-independent noise have been pointed out in $[16,17]$. The rationale of working in the undecimated wavelet domain is that classical dyadic wavelet decompositions, characterized by iterated filtering and downsampling, make the estimation of signal and noise variances critical due to aliasing introduced by decimation.

This paper is organized as follows. Section 2 is devoted to a brief discussion on speckle noise models. In Section 3, the LMMSE estimator and its application to despeckling will be introduced. After a brief review of the wavelet decomposition, the extension of LMMSE filtering in the wavelet domain will be presented. In Section 4, several experimental results will allow us to show the effectiveness of the proposed technique. Some concluding remarks are given in Section 5.

\section{SPECKLE MODELING}

The basic assumption behind the models of speckle noise is that the received signal from a specific resolution cell can be considered as the composition of several different phasors, having random, statistically independent, amplitudes and phases. Due to the large number of independent components, the received signal has a complex Gaussian distribution. A two-dimensional histogram of the detected complex image $z=x+j y$ is shown in Figure 1. The histogram has been computed from the pixels belonging to a homogeneous area of a tissue.

Let $a=\sqrt{x^{2}+y^{2}}$ be the amplitude of the acquired signal; it represents the signal that is finally displayed. The distribution of $a$ depends on the characteristics of the imaged

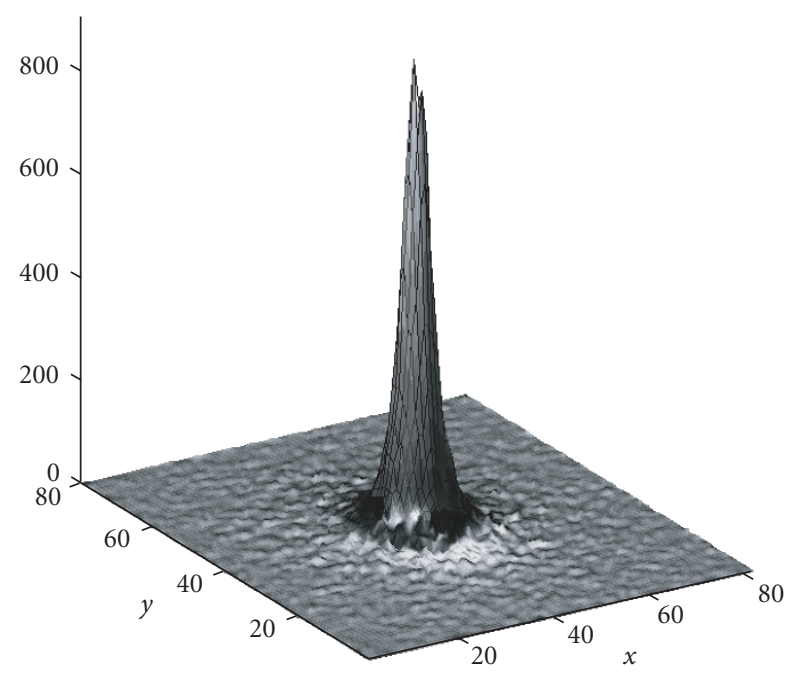

FIGURE 1: Two-dimensional histogram of the detected complex signal relative to a homogeneous area.

tissue. If the scattering structure is fine, the scattering surface is rough with respect to the wavelength, and the number of scatterers within a resolution cell is large, then the magnitude has a Rayleigh distribution; in this case, speckle noise is referred to as fully developed. Instead, if the backscattered signal can be modeled as a specular reflection, then the distribution is Rician $[18,19]$.

The first-order statistics of the Rayleigh distribution are

$$
\begin{gathered}
E[a]=\sqrt{\frac{\pi \sigma^{2}}{2}}, \\
\sigma_{a}^{2}=E\left[a^{2}\right]-E[a]^{2}=\sigma^{2} \frac{4-\pi}{2}
\end{gathered}
$$

and, consequently, defining the speckle contrast $c$ as the ratio between the standard deviation and the mean, we have

$$
c=\frac{\sqrt{\sigma_{a}^{2}}}{E[a]}=\sqrt{\frac{4-\pi}{\pi}}=0.52 .
$$

This property of the Rayleigh distribution suggests that speckle has a multiplicative nature that leads to bind together the local value of the image with the standard deviation of the noise term. Similar multiplicative noise models have been proposed in the literature to deal with coherent acquisition systems different than ultrasonic scanners [20,21].

In this paper, a general multiplicative noise model is used. For the sake of simplicity, consider a one-dimensional signal. The observed signal $g(n)$ is expressed by

$$
g(n)=f(n)+v(n)=f(n)+f(n)^{\gamma} \cdot u(n),
$$

in which $f(n)$ is the original, or noise-free, signal. The noisegenerating random process $u(n)$ is assumed independent of $f(n)$, stationary, uncorrelated, with zero mean and variance $\sigma_{u}^{2}$. The term $v(n)=f^{\gamma}(n) \cdot u(n)$ represents an additive 
signal-dependent noise. Since $f(n)$, in general, is not stationary, the noise term $v(n)$ must be assumed as nonstationary as well. The quantity $\gamma$ acts as a parameter that generalizes the noise model. The values of $\gamma$ that are considered in the literature are essentially two. The value $\gamma=1$ yields a purely multiplicative noise model. This model is accepted not only for ultrasonic scanners [22] but also for synthetic aperture radar (SAR) images [23]. However, in the ultrasound community, also the value $\gamma=0.5$ is taken into consideration, especially to model speckle noise of images where a logarithmic precompression has been performed $[10,24]$. Therefore, both these cases will be considered hereafter.

\section{FILTERING SPECKLE NOISE}

\subsection{LLMMSE filtering}

The processes introduced in (3) can be represented as vectors and will be indicated by $\mathbf{f}, \mathbf{g}$, and $\mathbf{v}$, where $\mathbf{f}$ is the noise-free signal, $\mathbf{g}$ is the observed signal, and $\mathbf{v}$ is the noise term. In this section, we still consider one-dimensional signals. The extension to the two-dimensional signal case is straightforward. The MMSE estimate of $\mathbf{f}$ is its expectation conditional to the observed signal, that is, $\hat{\mathbf{f}}_{\mathrm{MMSE}}=E[\mathbf{f} \mid \mathbf{g}]$. This is usually too complex to be computed, so we resort to the LMMSE estimator, that requires only statistics up to the second order and is given by,

$$
\hat{\mathbf{f}}_{\mathrm{LMMSE}}=E[\mathbf{f}]+\mathbf{C}_{\mathrm{fg}} \mathbf{C}_{\mathbf{g}}^{-1} \cdot[\mathbf{g}-E(\mathbf{g})],
$$

[25], where the matrices $\mathbf{C}_{\mathrm{g}}$ and $\mathbf{C}_{\mathrm{fg}}$ are the covariance of $\mathbf{g}$ and the cross-covariance between $\mathbf{f}$ and $\mathbf{g}$, respectively. Equation (4) imposes a global MSE minimization over the whole image within the constraint of a linear solution. The solution is optimum if the joint pdf's of $\mathbf{f}$ are multivariate Gaussian. Suppose now that $\mathbf{f}$ is uncorrelated, that is, $\mathbf{C}_{\mathbf{f}}=E\left\{[\mathbf{f}-E(\mathbf{f})][\mathbf{f}-E(\mathbf{f})]^{T}\right\}$ is a diagonal matrix. This fact means that all the correlation of $\mathbf{f}$ is conveyed by its space-varying mean $E[\mathbf{f}]$ only. Let $\sigma_{f}^{2}(n)$ and $\sigma_{g}^{2}(n)$ denote the variances of $\mathbf{f}$ and $\mathbf{g}$ at the $n$th sample position. It can be shown that [26], under the assumed hypotheses, the covariance matrices $\mathrm{C}_{\mathrm{g}}$ and $\mathrm{C}_{\mathrm{fg}}$ are diagonal and are given by $\mathbf{C}_{\mathbf{g}}=\operatorname{diag}\left[\sigma_{g}^{2}(1), \sigma_{g}^{2}(2), \ldots, \sigma_{g}^{2}(N)\right]$ and $\mathbf{C}_{\mathrm{fg}}=\mathbf{C}_{\mathbf{f}}=$ $\operatorname{diag}\left[\sigma_{f}^{2}(1), \sigma_{f}^{2}(2), \ldots, \sigma_{f}^{2}(N)\right]$. By replacing these functions into (4), we can see that the estimate of $\mathbf{f}$ is a pointwise operator. The local LMMSE (LLMMSE) estimate of $f(n)$ is given by (see [26])

$$
\hat{f}_{\text {LLMMSE }}(n)=E[f(n)]+\frac{\sigma_{f}^{2}(n)}{\sigma_{g}^{2}(n)} \cdot(g(n)-E[g(n)]) .
$$

To apply the filter in (5), we need to know $E[f]$ and $\sigma_{f}^{2}$. From the model in (3), since $u(n)$ is assumed zero mean and independent of $f(n)$, we have

$$
E[g(n)]=E[f(n)] .
$$

The variance of the observed signal can be expressed as

$$
\begin{aligned}
\sigma_{g}^{2}(n) & =E\left[g^{2}(n)\right]-E[g(n)]^{2} \\
& =E\left[f^{2}(n)+f(n)^{2 \gamma} u^{2}(n)+2 f(n)^{\gamma+1} u(n)\right]-E[f(n)]^{2} \\
& =\sigma_{f}^{2}(n)+\sigma_{u}^{2} f_{\gamma}(n) .
\end{aligned}
$$

The term $f_{\gamma}(n)=E\left[f^{2 \gamma}(n)\right]$ is dependent on the model we assume for the speckled image. For the models we consider in this paper, this term becomes

$$
f_{\gamma}(n)= \begin{cases}E[f(n)], & \gamma=0.5 \\ E\left[f^{2}(n)\right], & \gamma=1 .\end{cases}
$$

Hence, $f_{\gamma}(n)$ can be estimated from first-order statistics of the noise-free image.

Substituting expression (8) into (7) allows us to estimate the variance of the original image as

$$
\sigma_{f}^{2}(n)= \begin{cases}\sigma_{g}^{2}(n)-\sigma_{u}^{2} E[g(n)], & \gamma=0.5, \\ \frac{\sigma_{g}^{2}(n)-E[g(n)]^{2} \sigma_{u}^{2}}{1+\sigma_{u}^{2}}, & \gamma=1,\end{cases}
$$

where we have used (6). Hence, the final expression for the LLMMSE estimator in (5) may be rewritten as

$$
\hat{f}_{\mathrm{LLMMSE}}(n)=\left\{\begin{array}{c}
E[g(n)]+\frac{\sigma_{g}^{2}(n)-\sigma_{u}^{2} E[g(n)]}{\sigma_{g}^{2}(n)} \\
\cdot(g(n)-E[g(n)]), \quad \gamma=0.5, \\
E[g(n)]+\frac{\sigma_{g}^{2}(n)-E[g(n)]^{2} \sigma_{u}^{2}}{\left(1+\sigma_{u}^{2}\right) \sigma_{g}^{2}(n)} \\
\cdot(g(n)-E[g(n)]), \quad \gamma=1 .
\end{array}\right.
$$

The LLMMSE estimate uses only the first-order statistics of the observed image. The estimator $\hat{f}_{\text {LLMMSE }}(n)$ can be reformulated introducing local approximations of the nonstationary mean and variance of the observed image calculated as

$$
\begin{aligned}
E[g(n)] & \cong \bar{g}(n)=\frac{1}{2 W+1} \sum_{i=-W}^{W} g(n+i), \\
\sigma_{g}^{2}(n) & \cong \frac{1}{2 W} \sum_{i=-W}^{W}(g(n+i)-\bar{g}(n))^{2},
\end{aligned}
$$

where $2 W+1$ is the size of the local window. To avoid the numerator in (10) to be negative, it is clipped to positive values after the substitution of (11).

In Section 3.2, we show the application of the LLMMSE algorithm in the wavelet domain. The experimental results will demonstrate that filtering in the wavelet domain largely improves the performance of the method in terms of both texture preservation and homogeneous areas smoothing. 


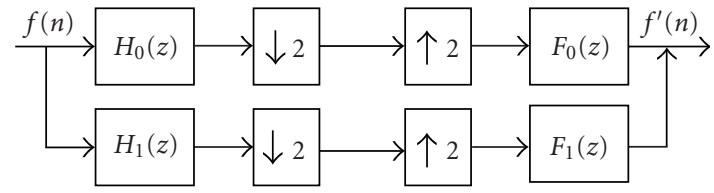

(a)

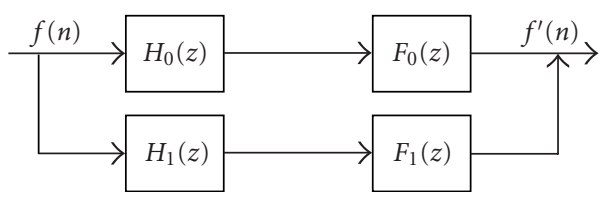

(b)

FIGURE 2: (a) Scheme of a critically sampled wavelet decomposition; (b) scheme with undecimated wavelet subbands.

\subsection{LLMMSE filtering in the wavelet domain}

Wavelet analysis provides a multiresolution representation of continuous and discrete-time signals and images $[27,28]$.

For discrete-time signals, the wavelet decomposition is implemented filtering the input signal with a lowpass filter $H_{0}(z)$ and a highpass filter $H_{1}(z)$, and downsampling the outputs by a factor of 2 . The two output sequences represent a smoothed version of $f(n)$, or approximation, and the rapid changes occurring within the signal, or detail. To achieve signal reconstruction, the coefficients of the approximation and detail signals are upsampled and filtered by a lowpass and a highpass filter, $F_{0}(z)$ and $F_{1}(z)$, respectively. The scheme of a wavelet decomposition and reconstruction is depicted in Figure 2a, in which $f(n)$ is a discrete 1D sequence and $f^{\prime}(n)$ the sequence reconstructed after the analysis/synthesis stages. As it can be seen, the wavelet representation is closely related to a subband decomposition scheme [29]. A two-channel representation can also be obtained, eliminating the downsampling and upsampling blocks and yielding the scheme shown in Figure 2b. It can be shown that, thanks to wavelet filters properties, perfect reconstruction of the input signal is maintained. Using an undecimated wavelet will allow us to simplify the representation of signal and noise in the transformed domain.

Applying the same splitting to the lowpass channel of a wavelet decomposition yields a two-level wavelet transform, whose scheme is shown in Figure 3a. Extending the scheme to $K$ levels of decomposition is straightforward. We use the notation $f_{k}^{(l)}(n)$ and $f_{k}^{(h)}(n)$ to denote the lowpass and highpass wavelet coefficients at the $k$ th level of the decomposition, respectively.

An equivalent representation of the two-level analysis bank is given in Figure 3b. It is obtained from that of Figure 3a by shifting the downsamplers towards the output of the system and by using upsampled filters, as noble identities state [29]. As can be seen, the wavelet coefficients $f_{k}^{(l)}(n)$

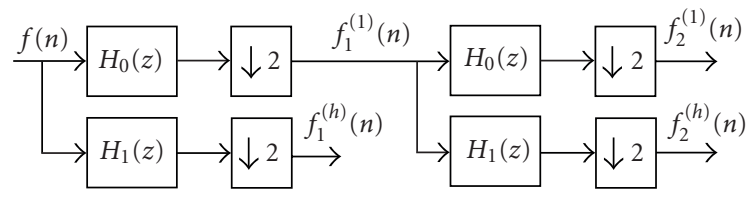

(a)

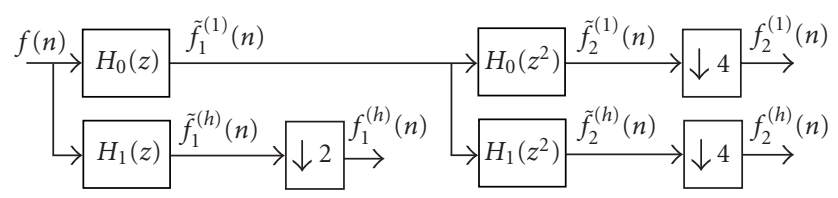

(b)

Figure 3: (a) Scheme of a two-level critically sampled wavelet decomposition; (b) equivalent scheme with undecimated wavelet subbands (denoted with a tilde).

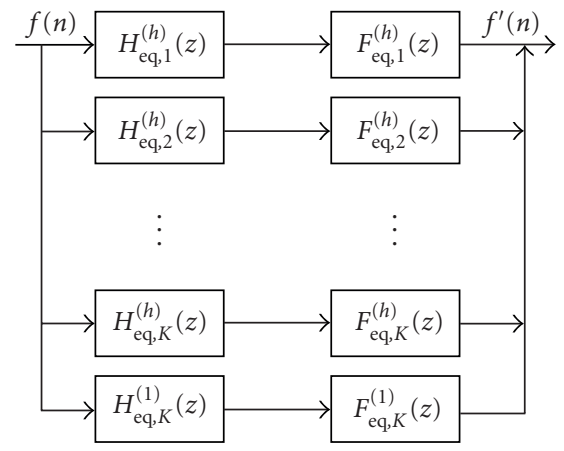

Figure 4: Equivalent scheme for a $K$-level undecimated wavelet.

and $f_{k}^{(h)}(n)$ can be obtained from the undecimated outputs $\tilde{f}_{k}^{(l)}(n)$ and $\tilde{f}_{k}^{(h)}(n)$, that will be referred to as undecimated wavelet coefficients. It can be easily noted that the sequences $\tilde{f}_{k}^{(l)}(n)$ and $\tilde{f}_{k}^{(h)}(n)$ are obtained by filtering the original signal with equivalent filters whose expressions are

$$
\begin{aligned}
& H_{\mathrm{eq}, k}^{(l)}(z)=\prod_{m=0}^{k-1} H_{0}\left(z^{2^{m}}\right), \\
& H_{\mathrm{eq}, k}^{(h)}(z)=\left[\prod_{m=0}^{k-2} H_{0}\left(z^{2^{m}}\right)\right] \cdot H_{1}\left(z^{2^{k-1}}\right) .
\end{aligned}
$$

It can be easily shown that perfect reconstruction can be obtained by dropping downsamplers and upsamplers from the $K$-level analysis/synthesis scheme. The equivalent $K$-level undecimated wavelet representation is shown in Figure 4.

Consider now the representation of signal and noise in the undecimated wavelet domain. The projection of a signal is obtained by filtering it with either $h_{\mathrm{eq}, k}^{(l)}(n)$ or $h_{\mathrm{eq}, k}^{(h)}(n)$. Due 
to the linearity of the transform, we have

$$
\begin{aligned}
\tilde{g}_{k}^{(l)}(n) & =f(n) * h_{\mathrm{eq}, k}^{(l)}(n)+v(n) * h_{\mathrm{eq}, k}^{(l)}(n) \\
& =\tilde{f}_{k}^{(l)}(n)+\tilde{v}_{k}^{(l)}(n), \\
\tilde{g}_{k}^{(h)}(n) & =f(n) * h_{\mathrm{eq}, k}^{(h)}(n)+v(n) * h_{\mathrm{eq}, k}^{(h)}(n) \\
& =\tilde{f}_{k}^{(h)}(n)+\tilde{v}_{k}^{(h)}(n) .
\end{aligned}
$$

Without loss of generality, we refer to the highpass and bandpass wavelet coefficients and, for the sake of simplicity, we drop the superscript $(h)$.

Now, we would like to apply the LLMMSE estimation algorithm to the signals obtained from the undecimated wavelet decomposition. Hence, we need their first-order statistics. The mean of the noise component is given by

$$
\begin{aligned}
E\left[\tilde{v}_{k}(n)\right] & =E\left[\sum_{i} h_{\mathrm{eq}, k}(i) f^{\gamma}(n-i) u(n-i)\right] \\
& =\sum_{i} h_{\mathrm{eq}, k}(i) E\left[f^{\gamma}(n-i)\right] E[u(n-i)]=0 .
\end{aligned}
$$

Therefore, we have

$$
E\left[\tilde{g}_{k}(n)\right]=E\left[\tilde{f}_{k}(n)\right]
$$

The estimate in (5) needs the knowledge of the variances of $\tilde{g}_{k}(n)$ and $\tilde{f}_{k}(n)$, or equivalently, of $E\left[\tilde{g}_{k}^{2}(n)\right]$ and $E\left[\tilde{f}_{k}^{2}(n)\right]$. The expression of $E\left[\tilde{g}_{k}^{2}(n)\right]$ is given by

$$
\begin{aligned}
E\left[\tilde{g}_{k}^{2}(n)\right]= & E\left[\left(\tilde{f}_{k}(n)+\tilde{v}_{k}(n)\right)^{2}\right] \\
= & E\left[\tilde{f}_{k}^{2}(n)\right]+E\left[\tilde{v}_{k}^{2}(n)\right] \\
& +2 \sum_{i} \sum_{j} h_{\mathrm{eq}, k}(i) h_{\mathrm{eq}, k}(j) \\
& \times E\left[f^{\gamma}(n-i) f^{\gamma}(n-j) u(n-j)\right] \\
= & E\left[\tilde{f}_{k}^{2}(n)\right]+E\left[\tilde{v}_{k}^{2}(n)\right],
\end{aligned}
$$

the double summation term being identically zero, thanks to the independence of $f$ and $u$. Therefore, by using (15), we have

$$
\sigma_{\tilde{f}_{k}}^{2}(n)=\sigma_{\tilde{g}_{k}}^{2}(n)-E\left[\tilde{v}_{k}^{2}(n)\right]
$$

where $E\left[\tilde{v}_{k}^{2}(n)\right]$ is given by

$$
\begin{aligned}
E\left[\tilde{v}_{k}^{2}(n)\right]=E\left[\sum_{i} \sum_{j} h_{\mathrm{eq}, k}(i) h_{\mathrm{eq}, k}(j)\right. \\
\left.\cdot f^{\gamma}(n-i) u(n-i) f^{\gamma}(n-j) u(n-j)\right] \\
=\sum_{i} h_{\mathrm{eq}, k}(i)^{2} \sigma_{u}^{2} \cdot E\left[f^{2 \gamma}(n-i)\right],
\end{aligned}
$$

where we used the uncorrelatedness of $u(n)$. The computation of $E\left[\tilde{v}_{k}^{2}(n)\right]$ is different according to the model we use, that is,

$$
E\left[\tilde{v}_{k}^{2}(n)\right]=\left\{\begin{array}{l}
\sum_{i} h_{\mathrm{eq}, k}(i)^{2} \sigma_{u}^{2} \cdot E[g(n-i)], \quad \gamma=0.5, \\
\sum_{i} h_{\mathrm{eq}, k}(i)^{2} \frac{\sigma_{u}^{2}}{1+\sigma_{u}^{2}} E\left[g(n-i)^{2}\right], \quad \gamma=1 .
\end{array}\right.
$$

For the case $\gamma=1$, we have used the fact that $E\left[g^{2}(n)\right]=$ $\left(1+\sigma_{u}^{2}\right) E\left[f^{2}(n)\right]$, which can be obtained by taking the squares of the model in (3) and exploiting the independence of $f$ and $u$.

Using (17) into the LLMMSE, estimator (5) yields

$$
\begin{aligned}
\hat{f}_{\text {LLMMSE }}(n)= & E\left[\tilde{g}_{k}(n)\right]+\frac{\sigma_{\tilde{g}_{k}}^{2}(n)-E\left[\tilde{v}_{k}^{2}(n)\right]}{\sigma_{\tilde{g}_{k}}^{2}(n)} \\
& \cdot\left(\tilde{g}_{k}(n)-E\left[\tilde{g}_{k}(n)\right]\right),
\end{aligned}
$$

where $E\left[\tilde{v}_{k}^{2}(n)\right]$ is given by (19). The LLMMSE estimate of the undecimated wavelet coefficients is computable by using the observed first-order statistics of $\tilde{g}_{k}(n)$ as well as $E[g(n)]$ and $\sigma_{g}^{2}(n)$. All these quantities can be computed as local averages. Actually, for detail signals, the function $E\left[\tilde{g}_{k}^{(h)}(n)\right]$ may be assumed to be approximately zero, thus simplifying the estimator.

After the denoised wavelet coefficients have been estimated, the restored signal is to be reconstructed. A first possibility is to use a classical wavelet scheme, in which the critically sampled wavelet coefficients are reconstructed by using upsampling and synthesis filters. A second possibility is dropping the downsamplers and upsamplers and using the scheme shown in Figure 4.

\section{EXPERIMENTAL RESULTS}

The performance of the proposed method has been assessed by using both images affected by synthetic speckle and actual ultrasonic images.

In order to evaluate quantitatively the performance of the algorithm, we used images corrupted by synthetic noise. The noise model in (3) with $\gamma=0.5$ and $\gamma=1$ has been used. We have compared the spatial LLMMSE algorithm, proposed for $\gamma=1$ in [26], and denoted hereafter as Kuan filter, with its multiscale version denoted as undecimated wavelet scaling (UWS). The test image Lenna has been corrupted by a speckle pattern characterized by values of $\gamma$ equal to either 0.5 or 1 . Raw images with $\mathrm{SNR}=2.9 \mathrm{~dB}$ and $\mathrm{SNR}=9.9 \mathrm{~dB}$ have been processed. Table 1 shows the SNR obtained after using the Kuan filter and UWS algorithm. As can be seen, UWS outperforms Kuan filter of about 2-3 dB. In Figure 5, visual results show that UWS yields better performances in terms of both speckle removal and image contrast enhancements with respect to Kuan filter.

Ultrasonic images have been acquired with two different probes. In particular, an image of a human liver has been 


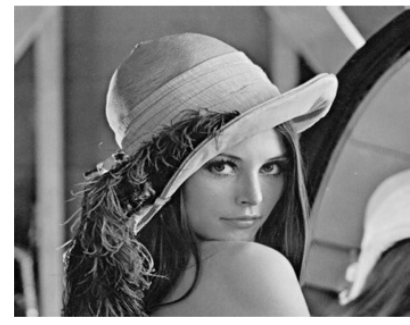

(a)

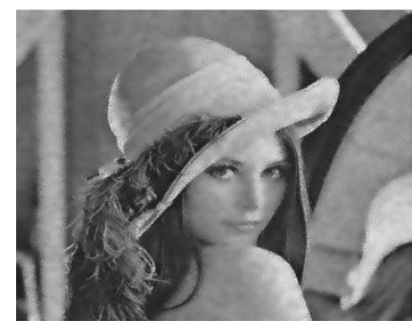

(c)

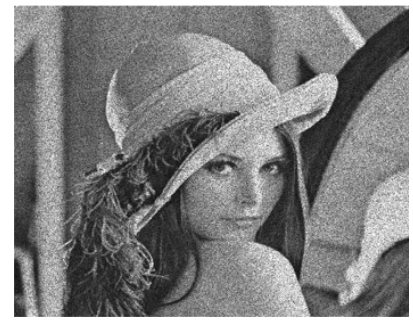

(b)

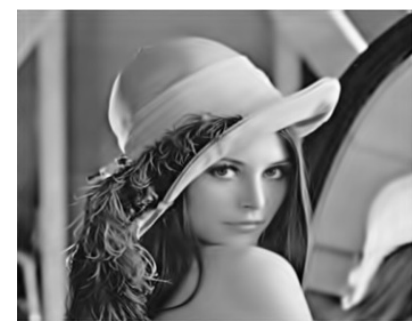

(d)

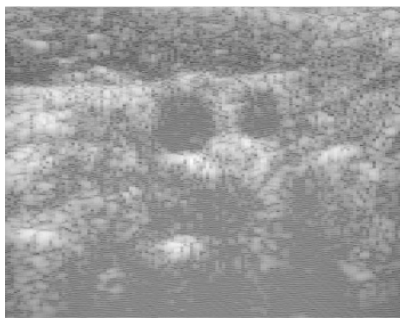

(a)

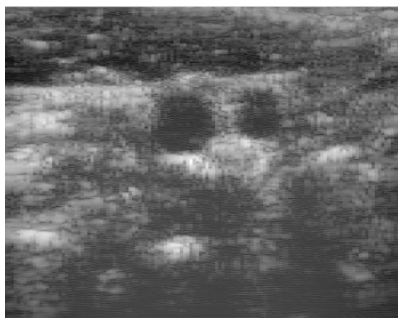

(c)

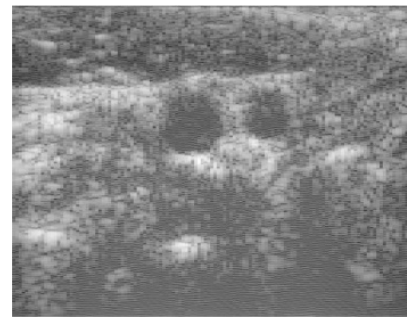

(b)

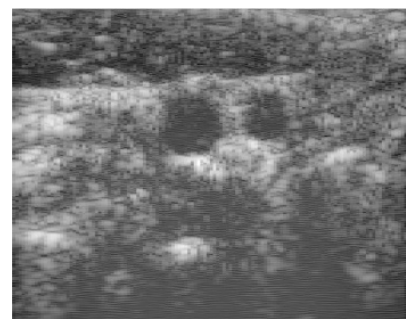

(d)

Figure 5: (a) Original image Lenna. (b) Speckled image corrupted with $\gamma=1$ and SNR $=2.9 \mathrm{~dB}$. (c) Image obtained by filtering with Kuan's algorithm. (d) Image obtained by filtering with UWS algorithm.

TABLE 1: SNR (dB) of denoised images. Raw image with either $\mathrm{SNR}=2.9 \mathrm{~dB}$ or $\mathrm{SNR}=9.9 \mathrm{~dB}$. Noise generated and filtered assuming $\gamma=0.5$ and $\gamma=1$.

\begin{tabular}{ccccc}
\hline \multirow{2}{*}{ Filter } & \multicolumn{2}{c}{ SNR $=2.9 \mathrm{~dB}$} & \multicolumn{2}{c}{ SNR $=9.9 \mathrm{~dB}$} \\
& $\gamma=0.5$ & $\gamma=1$ & $\gamma=0.5$ & $\gamma=1$ \\
\hline Kuan & 11.9 & 12.3 & 15.5 & 15.9 \\
UWS & 14.1 & 15.7 & 18.6 & 19.2 \\
\hline
\end{tabular}

generated by using a $5-\mathrm{MHz}$ probe, and an image of the carotid artery bifurcation has been generated by using a 7.5$\mathrm{MHz}$ probe. Both of these probes are phased arrays of 128 elements. The focus has been set at a distance of around 5 centimeters from the probe-tissue interface. After envelope detection and logarithmic compression, the images look like as they appear in Figures 6a and 7a. The two darker circles appearing almost in the center of Figure $6 \mathrm{a}$ are the transverse sections of the carotid artery bifurcation. In this image, the main effect of speckle is to reduce the contrast around the borders of the arteries, highlighting no significative structures in low-level signal regions. Moreover, it tends to mask the real structure of the tissues surrounding the vessels. The scanning of the liver is visible in the bottom area of Figure $7 \mathrm{a}$. Particularly interesting interfaces of the abdominal tissues are visible in the zone near the probe, positioned at the top of the figure. Speckle noise effects are evident in the region representing the liver, where a strong granular pattern is superimposed to the characteristic texture of the liver.

FIgURE 6: (a) Ultrasonic image of a carotid artery bifurcation. (b) Results of filtering obtained with frequency compounding. (c) Kuan filter. (d) UWS filter.

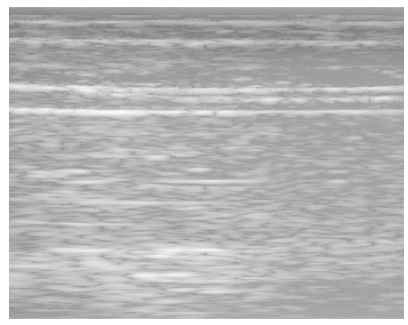

(a)

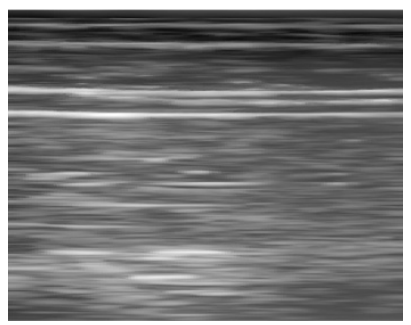

(c)

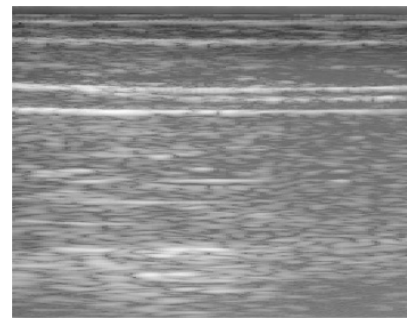

(b)

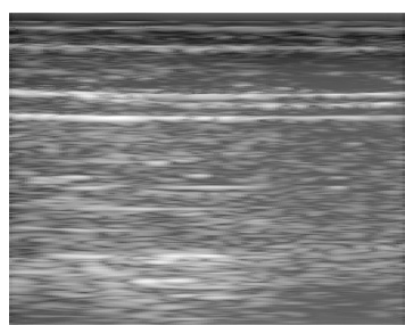

(d)
FIGURE 7: (a) Ultrasonic image of the liver and some abdominal interfaces. (b) Results of filtering obtained with frequency compounding. (c) Kuan filter. (d) UWS filter.

TABLE 2: Estimated noise model parameters.

\begin{tabular}{lcc}
\hline Image & $\gamma$ & $\sigma_{u}$ \\
\hline Liver & 0.965 & 0.485 \\
Carotid & 1.079 & 0.311 \\
\hline
\end{tabular}




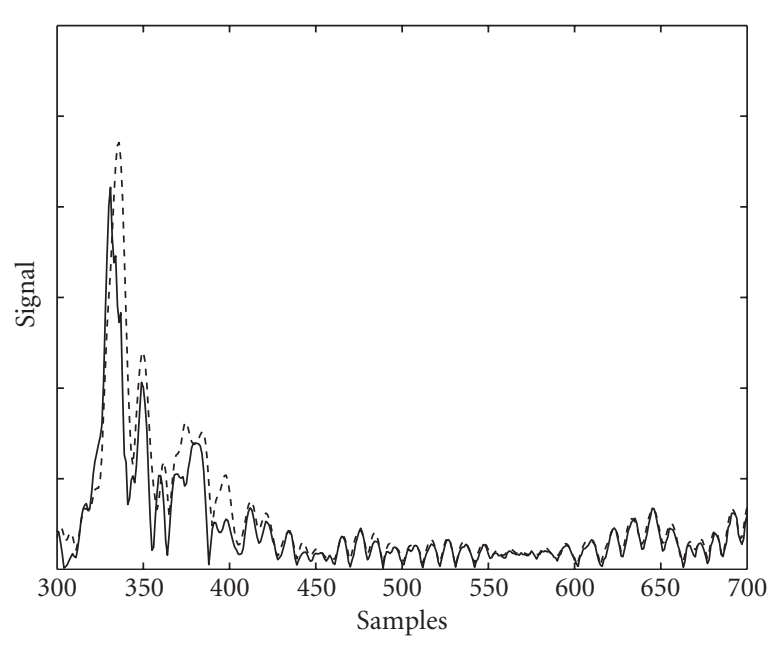

(a)

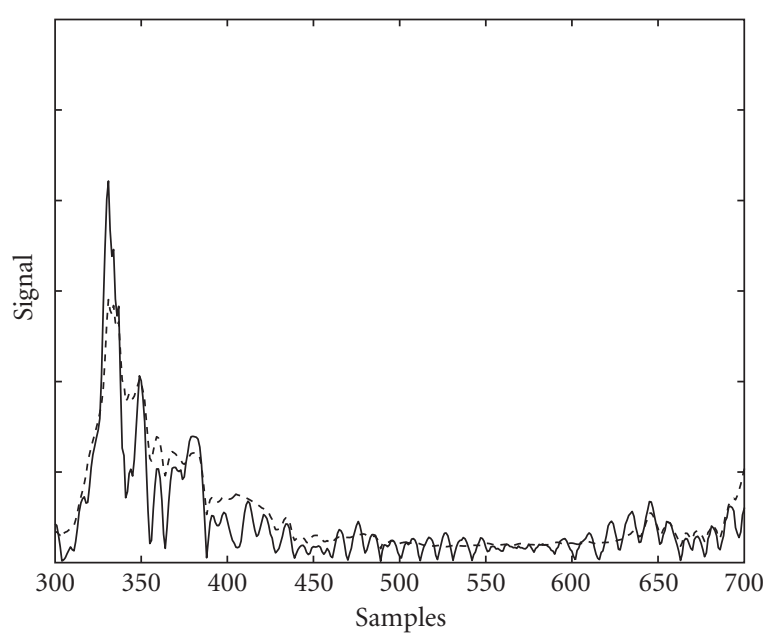

(b)

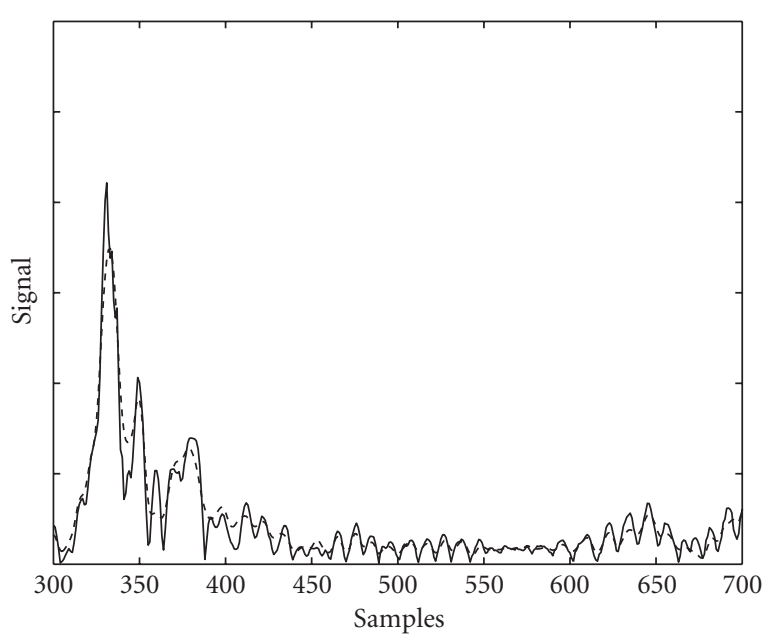

(c)

FIGURE 8: Filtering of a single trace. Original trace (solid line) compared to the filtered traces (dashed lines) obtained with (a) frequency compound, (b) Kuan filter, and (c) UWS algorithm.
Three bands frequency compounding has been applied to the acquired signals for a comparison with the proposed denoising methods. Results obtained by applying this technique are shown in Figures $6 \mathrm{~b}$ and $7 \mathrm{~b}$. Frequency compounding improves the image quality, removing part of the granular speckle pattern that corrupts the original texture of the tissue, at the price of a reduction of resolution in the longitudinal direction. However, speckle still affects darker areas.

Both ultrasonic images have been processed, after envelope detection, with the filters proposed in this paper. The knowledge of the variance $\sigma_{u}^{2}$ of the noise generator process and of the parameter $\gamma$ is needed to filter the images. A procedure to estimate $\sigma_{u}^{2}$ and $\gamma$ has been proposed in [30]. The method is based on the fact that, in homogeneous areas, $\log \sigma_{g}$ is a linear function of $\log E[g]$, where the linear function parameters are dependent on $\gamma$ and $\sigma_{u}^{2}$. Hence, linearbest fitting of measured data yields their estimates. The estimated parameters $\gamma$ and $\sigma_{u}^{2}$ for the acquired images are shown in Table 2. These results reveal that the actual value of $\gamma$ for our imaging system is approximately equal to 1 . Thus, the case $\gamma=0.5$ will not be considered for actually acquired images.

The scanned signals have been processed with Kuan filter and UWS after envelope detection and before logarithmic compression. Applying Kuan filter yields the results shown in Figures $6 \mathrm{c}$ and $7 \mathrm{c}$. In this case, less speckle is smoothed out with respect to frequency compounding both in low- and high-level areas. The results obtained with the UWS algorithm are displayed in Figures $6 \mathrm{~d}$ and $7 \mathrm{~d}$. The filtered image reveals the real structure of tissues, preserving the sharpness of edges and without loss of resolution.

In order to betterly understand the behavior of each different filter, a single trace of the ultrasonic scanner and its filtered versions are shown in Figure 8. The signal is a portion of a trace belonging to the scan of the liver. The position of the several interfaces can be detected as peaks in the trace. Frequency compound, Kuan filter, and UWS algorithm have been applied. Results show that frequency compound seems to preserve most of the edges of reflectors and to remove speckle noise. Kuan filter tends to destroy strong reflectors peaks and smooth out small structures. The UWS algorithm preserves peak positions and sharpness as well as smoothes low-level signal regions.

\section{CONCLUSIONS}

In this paper, a procedure to denoise images affected by additive signal-dependent noise has been proposed. The method relies on the knowledge of a general parametric model for the additive noise and uses LLMMSE estimation in an undecimated wavelet domain. The proposed method has been tested on both synthetically speckled images and ultrasonic images. Experimental results of the proposed method demonstrate an efficient rejection of the distortion due to speckle with respect to other commonly used noise reduction techniques. 


\section{ACKNOWLEDGMENTS}

The authors would like to thank the anonymous reviewers whose comments and suggestions have helped in improving the quality of the original manuscript. The authors would also like to thank Prof. Leonardo Masotti and Prof. Elena Biagi for allowing them to use the Fast Echographic Multiparametric Multi-Imaging Novel Apparatus (FEMMINA) platform for the acquisition and the representation of the ultrasonic images used in this work.

\section{REFERENCES}

[1] J. W. Goodman, Statistical Optics, Wiley-Interscience, New York, NY, USA, 1985.

[2] M. O’Donnell and S. W. Flax, "Phase aberration measurements in medical ultrasound: human studies," Ultrasonic Imaging, vol. 10, no. 1, pp. 1-11, 1988.

[3] G. E. Trahey, P. D. Freiburger, L. F. Nock, and D. C. Sullivan, "In vivo measurements of ultrasonic beam distortion in the breast," Ultrasonic Imaging, vol. 13, no. 1, pp. 71-90, 1991.

[4] L. M. Hinkelman, D. L. Liu, L. A. Metlay, and R. C. Waag, "Measurement of ultrasonic pulse arrival time and energy level variations produced by propagation through abdominal wall," Journal of the Acoustical Society of America, vol. 95, no. 1, pp. 530-541, 1994.

[5] L. M. Hinkelman, D. L. Liu, R. C. Waag, Q. Zhu, and B. D. Steinberg, "Measurement and correction of ultrasonic pulse distortion produced by the human breast," Journal of the Acoustical Society of America, vol. 97, no. 3, pp. 1958-1969, 1995.

[6] P. A. Magnin, O. T. von Ramm, and F. L. Thurstone, "Frequency compounding for speckle contrast reduction in phased array images," Ultrasonic Imaging, vol. 4, no. 3, pp. 267-281, 1982.

[7] M. Berson, A. Roncin, and L. Pourcelot, "Compound scanning with an electrically steered beam," Ultrasonic Imaging, , no. 3, pp. 303-308, 1981.

[8] R. L. Galloway, B. A. McDermott, and F. L. Thurstone, "A frequency diversity process for speckle reduction in real-time ultrasonic images," IEEE Transactions on Ultrasonics, Ferroelectrics, and Frequency, vol. 35, no. 1, pp. 45-49, 1988.

[9] K. H. Uslu, N. Bilgutay, R. Murthy, and K. Kaya, "Medical image enhancement using split spectrum processing," in Proc. IEEE International Ultrasonic Symposium, vol. 2, pp. 993-997, Baltimore, Md, USA, 1993.

[10] T. Loupas, W. N. McDicken, and P. L. Allan, "An adaptive weighted median filter for speckle suppression in medical ultrasonic images," IEEE Trans. Circuits and Systems, vol. 36, no. 1, pp. 129-135, 1989.

[11] D. L. Donoho, "Denoising by soft-thresholding," IEEE Transactions on Information Theory, vol. 41, no. 3, pp. 613-627, 1995.

[12] M. C. Mihçak, I. Kozintsev, K. Ramchandran, and P. Moulin, "Low-complexity image denoising based on statistical modeling of wavelet coefficients," IEEE Signal Processing Letters, vol. 6, no. 12, pp. 300-303, 1999.

[13] S. P. Ghael, A. M. Sayeed, and R. G. Baraniuk, "Improved wavelet denoising via empirical Wiener filtering," in Proceedings of SPIE's 42nd Meeting, vol. 3169 of SPIE Proceedings, pp. 389-399, San Diego, Calif, USA, July 1997.

[14] S. G. Chang, B. Yu, and M. Vetterli, "Spatially adaptive wavelet thresholding with context modelling for image denoising," IEEE Trans. Image Processing, vol. 9, no. 9, pp. 1522-1531, 2000.
[15] R. Öktem, L. Yaroslavsky, and K. Egiazarian, "Transform domain approaches for image denoising," Journal of Electronic Imaging, vol. 11, no. 2, pp. 149-156, 2002.

[16] M. Lang, H. Guo, J. E. Odegard, C. S. Burrus, and R. O. Wells Jr., "Noise reduction using an undecimated discrete wavelet transform," IEEE Signal Processing Letters, vol. 3, no. 1, pp. 10-12, 1996.

[17] R. Coifman and D. L. Donoho, "Translation-invariant denoising," in Wavelets and Statistics, A. Antoniadis and G. Oppenheim, Eds., vol. 103 of Lecture Notes in Statistics, pp. 125150, Springer-Verlag, New York, NY, USA, 1995.

[18] C. B. Burckhardt, "Speckle in ultrasound B-mode scans," IEEE Transactions on Sonics and Ultrasonics, vol. 25, no. 1, pp. $1-6,1978$.

[19] R. F. Wagner, S. W. Smith, J. M. Sandrik, and H. Lopez, "Statistics of speckle in ultrasound B-scans," IEEE Transactions on Sonics and Ultrasonics, vol. 30, no. 3, pp. 156-163, 1983.

[20] W. K. Pratt, Digital Image Processing, John Wiley \& Sons, New York, NY, USA, 1991.

[21] A. K. Jain, Fundamentals of Digital Image Processing, Prentice Hall, Englewood Cliffs, NJ, USA, 1989.

[22] A. Achim, A. Bezerianos, and P. Tsakalides, "Novel Bayesian multiscale method for speckle removal in medical ultrasound images," IEEE Trans. on Medical Imaging, vol. 20, no. 8, pp. 772-783, 2001.

[23] M. Simard, G. DeGrandi, K. P. B. Thomson, and G. B. Bénié, "Analysis of speckle noise contribution on wavelet decomposition of SAR images," IEEE Transactions on Geoscience and Remote Sensing, vol. 36, no. 6, pp. 1953-1962, 1998.

[24] M. Karaman, M. A. Kutay, and G. Bozdagi, "An adaptive speckle suppression filter for medical ultrasonic imaging," IEEE Trans. on Medical Imaging, vol. 14, no. 2, pp. 283-293, 1995.

[25] S. M. Kay, Fundamentals of Statistical Processing, Volume I: Estimation Theory, Prentice Hall, Englewood Cliffs, NJ, USA, 1993.

[26] D. T. Kuan, A. A. Sawchuk, T. C. Strand, and P. Chavel, "Adaptive noise smoothing filter for images with signal-dependent noise," IEEE Trans. on Pattern Analysis and Machine Intelligence, vol. 7, no. 2, pp. 165-177, 1985.

[27] I. Daubechies, "Orthonormal bases of compactly supported wavelets," Commun. Pure Appl. Math., vol. 41, no. 7, pp. 909996, 1988.

[28] S. Mallat, "A theory for multiresolution signal decomposition: the wavelet representation," IEEE Trans. on Pattern Analysis and Machine Intelligence, vol. 11, no. 7, pp. 674-693, 1989.

[29] P. P. Vaidyanathan, Multirate Systems and Filter Banks, Prentice Hall, Englewood Cliffs, NJ, USA, 1992.

[30] G. Torricelli, F. Argenti, and L. Alparone, "Modelling and assessment of signal-dependent noise for image de-noising," in 11th European Conference on Signal Processing, EUSIPCO, pp. 287-290, Toulouse, France, September 2002.

Fabrizio Argenti obtained his "Laurea" degree cum laude in electronic engineering and the Ph.D. degree from the University of Florence, Italy, in 1989 and 1993, respectively. Since 1993, he has been with the Department of Electronics and Telecommunications of the University of Florence, first as an Assistant Professor, now as an Associate Professor of Digital Signal Processing and Telecommunications Systems. In 1992,

he was a Postgraduate Research Fellow at the Department of

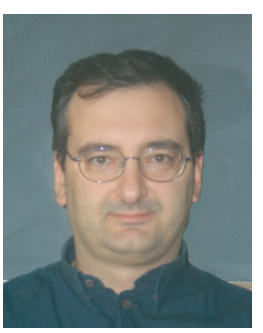


Electrical Engineering, University of Toronto, Canada. His main research interests are filter banks theory and design, wavelet theory and applications, audio compression, and multiresolution image analysis, processing, and fusion.

Gionatan Torricelli was born in Figline Valdarno (Florence), Italy, in 1976. He received the "Laurea" degree cum laude in electronic engineering in 2001. In 1999-2000, he attended four Master courses at the Computer Science Department of the Courant Institute, New York University. He is currently pursuing the Ph.D. degree at the Department of Electronics and Telecommunications at the University of Florence. His main

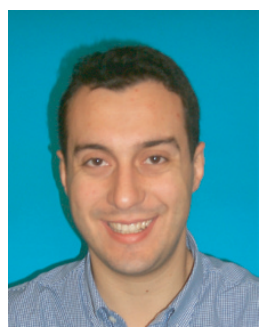
research interests are ultrasonic nondestructive evaluation, image processing, wavelet analysis, and denoising. 